\title{
Xenopus Paraxial Protocadherin regulates morphogenesis by antagonizing Sprouty
}

\author{
Yingqun Wang, ${ }^{1,4}$ Patricia Janicki, ${ }^{1}$ \\ Isabelle Köster, ${ }^{1}$ Corinna D. Berger, ${ }^{1}$ \\ Christian Wenzl, ${ }^{2}$ Jörg Großhans, ${ }^{2}$ \\ and Herbert Steinbeisser ${ }^{1,3}$
}

\begin{abstract}
${ }^{1}$ Institute of Human Genetics, University Heidelberg, 69120 Heidelberg, Germany; ${ }^{2}$ Center for Molecular Biology (ZMBH), University Heidelberg, 69120 Heidelberg, Germany
\end{abstract}

Xenopus Paraxial Protocadherin (xPAPC) has signaling functions that are essential for convergent extension (CE) movements and tissue separation during gastrulation. PAPC modulates components of the planar cell polarity (PCP) pathway, but it is not clear how PAPC is connected to $\beta$-catenin-independent Wnt-signaling. By yeast two-hybrid screen, we found that the intracellular domain of PAPC interacts with Sprouty (Spry), an inhibitor of CE movements. Upon binding to PAPC, Spry function is inhibited and PCP signaling is enhanced. Our data indicate that PAPC promotes gastrulation movements by sequestration of Spry and reveal a novel mechanism by which protocadherins modulate $\beta$-catenin-independent Wnt-signaling.

Supplemental material is available at http://www.genesdev.org.

Received August 14, 2007; revised version accepted January 18,2008

Paraxial Protocadherin (PAPC) was identified in Xenopus as a gene expressed in the Spemann organizer (Kim et al. 1998). Expression of xPAPC in the gastrula embryo is regulated by nodal, Wnt/ $\beta$-catenin and Wnt-5a signaling (Wessely et al. 2004; Schambony and Wedlich 2007). PAPC modulates in a yet unknown manner C-cadherinmediated cell adhesion through its extracellular domain and thereby promotes cell sorting (Chen and Gumbiner 2006). The intracellular domain of PAPC exerts signaling functions and is implicated in the regulation of convergent extension (CE) movements and separation behavior of the involuting mesoderm and the neuroectoderm (Kim et al. 1998; Medina et al. 2004; Unterseher et al. 2004). Zebrafish PAPC ( $z P A P C)$ gene is regulated by the transcription factor spadetail and $z P A P C$ function is required for $\mathrm{CE}$ movements in the fish gastrula (Yamamoto et al. 1998). In Xenopus, the regulation of CE movements and tissue separation by PAPC depends on

[Keywords: xPAPC; Sprouty; planar cell polarity; gastrulation movements]

Corresponding authors.

${ }^{3}$ E-MAIL Herbert.Steinbeisser@med.uni-heidelberg.de; FAX 6221-565153

${ }^{4}$ E-MAIL Yingqun.Wang@med.uni-heidelberg.de; FAX 6221-568884.

Article is online at http://www.genesdev.org/cgi/doi/10.1101/gad.452908. its ability to modulate the activity of Rho GTPase and c-jun N-terminal kinase, which are effectors of the planar cell polarity (PCP) pathway (Medina et al. 2004; Unterseher et al. 2004). However, the mechanism by which PAPC modulates PCP signaling remains unclear. Recently it was reported that Xenopus Sprouty 1 and 2 proteins act as inhibitors of the PCP pathway and are part of the morphogenetic machinery that regulates gastrulation (Sivak et al. 2005). In this study, we provide evidence that PAPC interacts with Sprouty and antagonizes its inhibitory effects on PCP, therefore providing novel insight into the link between protocadherin and PCP signaling.

\section{Results and Discussion}

In an effort to identify potential proteins involved in signaling downstream from xPAPC, we performed a yeast two-hybrid screen using the cytoplasmic domain of $\mathrm{xPAPC}(\mathrm{xPAPCc})$ as bait. Independent clones $\left(3.5 \times 10^{6}\right)$ of Xenopus laevis oocyte cDNA library were screened(Fig. 1A). Among the positive clones isolated was xSprouty1 (xSpry1). Sprouty is an inhibitor of receptor tyrosine kinase (RTK) signaling (Mason et al. 2006). In Drosophila, Sprouty (dSpry) inhibits FGF-mediated activation of mitogen-activated protein kinase (MAPK) in the larval trachea (Hacohen et al. 1998). Xenopus xSpry1 and $x$ Spry2, in contrast, do not inhibit MAPK-mediated transcription of FGF-target genes and do not interfere with mesoderm specification but instead block morphogenetic movements by interfering with PCP pathway (Nutt et al. 2001; Sivak et al. 2005).

dSpry as well as xSpry1 and xSpry2 interacted with xPAPCc (Fig. 1B). In contrast, xSpred1, a protein related to $\mathrm{xSpry1}$, failed to do so (Fig. 1B). Interestingly, Spred proteins inhibit MAPK signaling in Xenopus embryos but do not interfere with morphogenetic processes regulated by PCP pathway (Sivak et al. 2005). Mutations of putative phosphorylation sites in the XPAPCc peptide, which were identified by the Scansite computer program as putative 14-3-3-binding sites, weakened (S741A) or abolished (S955A) the interaction with xSpry1 (Fig. 1A,B). Amino acid exchanges that mimic phosphorylation did not impair xSpryl binding (S741E, S955E) (Fig. 1B). We have evidence that XPAPCc is phosphorylated in embryos and that the phosphorylation is reduced when S741 and S955 residues are mutated (Supplemental Fig. $\mathrm{S} 2 \mathrm{H})$.

Next, we set out to confirm the interaction of xPAPCc and xSpry1 in vivo by coimmunoprecipitation (co-IP) assays. Synthetic mRNAs for Myc-xSpry1, Flag-xPAPCc, and for the Flag-tagged intracellular domain of Xenopus Axial Protocadherin (Flag-xAXPCc) were injected into four-cell stage Xenopus embryos. Myc-xSpryl coimmunoprecipitated with Flag-xPAPCc but not with FlagxAXPCc using Flag antibody (Fig. 1C). Similarly, MycxSpry1 was not coimmunoprecipitated with FlagxPAPCc-S741A/S955A (Flag-xPAPCcmut) harboring point mutations (Fig. 1D). In the reciprocal experiment, Myc antibody specifically coimmunoprecipitated MycxSpry1 and Flag-xPAPCc but not Flag-xAXPCc or FlagxPAPCcmut (Fig. 1E; data not shown). These experiments corroborated the data obtained in the yeast two- 
A

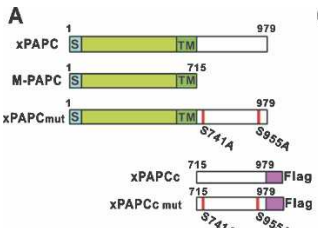

B

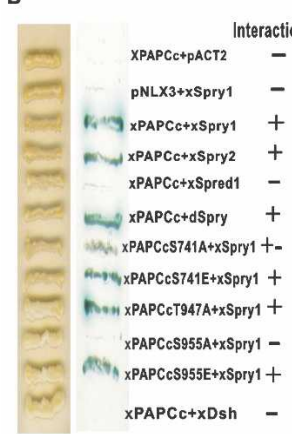

Leu Trp. LeUTrp His X-gal
C
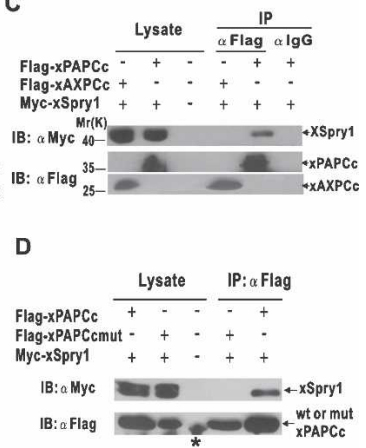

E

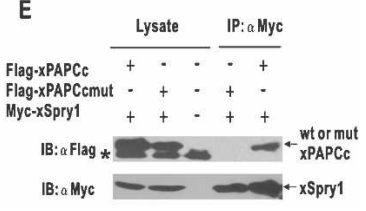

Figure 1. Physical interaction of xPAPC and Spry. (A) Schematic representation of Xenopus PAPC constructs used in this study. The signal peptide is marked in light blue, the transmembrane domain is marked in green, and the Flag tag is marked in pink. M-PAPC lacks the cytoplasmic domain and PAPCc lacks the extracellular and the transmembrane domains. In xPAPCmut and XPAPCcmut, both serine residues in 741 and 955 are mutated to alanine. (B) Yeast twohybrid assay. cDNAs were cloned into bait vector pNLX3 or prey vector pACT2 and cotransfected into yeast strain L40. One representative colony from each cotransformation was picked and streaked onto $\mathrm{Leu}^{-} \mathrm{Trp}^{-}$plate (left panel) or $\mathrm{Leu}^{-} \mathrm{Trp}^{-} \mathrm{His}^{-} \mathrm{X}-\mathrm{gal}^{+}$plate (right panel) and incubated for $3 \mathrm{~d}$ at $30^{\circ} \mathrm{C}$. Blue colonies are seen when bait and prey proteins interact. $(C-E)$ Four-cell-stage embryos were injected with Flag-xPAPCc, Flag-xPAPCcmut, or Flag-xAXPCc mRNA together with Myc-xSpry1 mRNA and grown to gastrula stages. Embryo lysates were subjected to co-IP and Western blot. Asterisks in $D$ and $E$ mark nonspecific bands.

hybrid assay and showed that xPAPC and xSpry1 specifically interact in Xenopus embryos.

As a consequence of this interaction, the subcellular localization of Spry should be changed from the cytoplasm to the membrane. When GFP-xSpryl was coexpressed with XPAPC in animal cap cells, reduced cytoplasmic and enhanced membrane staining was observed compared with cells that express GFP-xSpryl alone or in combination with M-PAPC, which lacks the intracellular domain (Fig. 2A). The membrane recruitment of GFPxSpryl by xPAPC was also confirmed in transfected HEK293 cells (Fig. 2B-E; Supplemental Fig. S2A-C). Like in animal cap cells, xPAPC recruited GFP-xSpry1 to the membrane. In contrast, a full-length PAPC construct harboring the S741A and S955A amino acid substitutions (xPAPCmut) was unable to promote membrane localization of GFP-xSpry1 (Fig. 2C). The ability of xPAPCmut to modulate cell adhesion and promote cell sorting, however, was not impaired (Supplemental Fig. S2D-G). Since in cultured mammalian cells membrane recruitment of Spry can be induced by the activation of FGF signaling (Hanafusa et al. 2002), we tested whether PAPC-mediated Spry recruitment to the membrane would occur when FGF signaling was blocked on the receptor level by the SU5402 inhibitor. SU5402 treatment did not impair membrane recruitment of xSpry1 by xPAPC but abolished bFGF-mediated xSpry1 recruitment (Fig. 2E; Supplemental Fig. S3). Our results in HEK cells showed that $\mathrm{xPAPC}$ is sufficient for the membrane recruitment of Spry and that PAPC/Spry interaction is not dependent on FGF signaling. Membrane recruitment of xSpry1 did not occur in the presence of xPAPCmut, demonstrating that this process is dependent on the interaction of Spry and xPAPC. xPAPC-mediated membrane localization of xSpryl in Xenopus animal cap tissue and in HEK293 cells suggests an interaction of these two proteins in vivo.

Next, we addressed the biological significance of this interaction. $x P A P C$ and $x$ Spry 1 share similar expression patterns during gastrulation (Kim et al. 1998; Sivak et al. 2005). In situ hybridization analysis on hemisectioned embryos at the early gastrulation stage showed that the expression domains of $x P A P C$ and $x$ Spry 1 overlap in the dorsal marginal zone (DMZ) (Supplemental Fig. S1). Since $x$ Spry is an inhibitor of the PCP pathway and $\mathrm{xPAPC}$ is a positive regulator of PCP signaling, we propose that xPAPC could modulate PCP by specifically
A

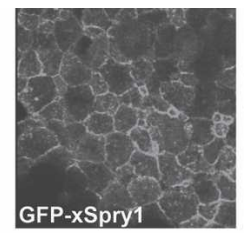

B

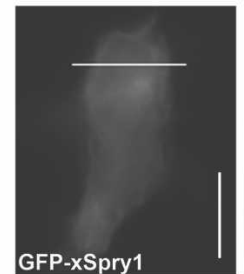

C

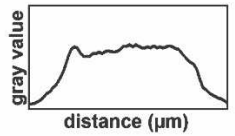

D
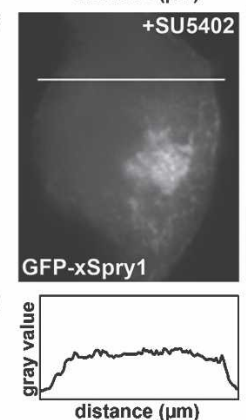
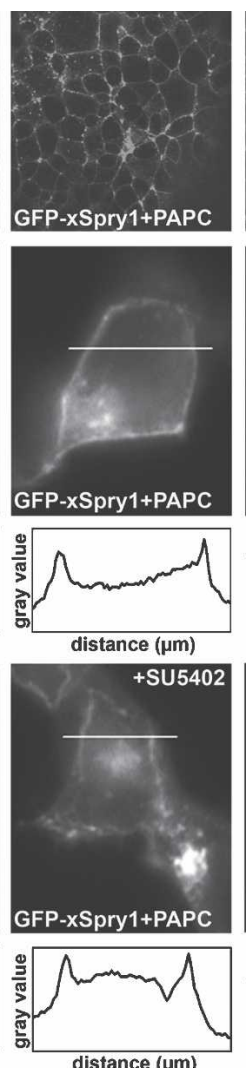
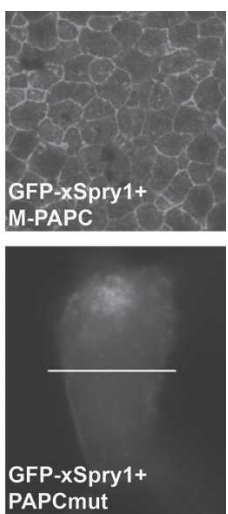

Figure 2. $x P A P C$ recruits $x$ Spryl to the cell membrane. $(A)$ Membrane recruitment of GFP-xSpryl by xPAPC in animal cap cells. GFP-xSpry1 mRNA (400 pg) was injected alone or with mRNAs encoding xPAPC (600 pg) or M-PAPC (600 pg) into four-cell-stage embryos. Animal caps were excised at stage 9, and the localization of GFP-xSpryl was determined by confocal microscopy. $(B) \mathrm{Mem}-$ brane recruitment of GFP-xSpry1 by xPAPC in HEK293 cells. Cells were transfected with GFP-xSpryl alone or in combination with xPAPC or xPAPCmut and the localization of GFP-xSpryl was determined by fluorescent microscopy. $(C)$ Two-dimensional plot profile of pixel intensity along the white lines in $B$ using the ImageJ program. $(D)$ xPAPC recruits GFP-xSpryl to the cell membrane in the absence of FGF signaling: cells expressing GFP-xSpry1 alone or in combination with XPAPC or xPAPCmut in the presence of $5 \mu \mathrm{M}$ SU5402. (E) Two-dimensional plot profile of pixel intensity along the white lines in $D$. Bar, $10 \mu \mathrm{m}$. 
antagonizing xSpryl. xPAPC expression is induced in animal caps exposed to the TGF- $\beta$ growth factor activin (Hukriede et al. 2003; Chen and Gumbiner 2006), and such explants elongate due to PCP-dependent CE movements (Kim et al. 1998; Chen and Gumbiner 2006). Activin-induced elongation $(95 \%, n=20)$ (Fig. 3A) was inhibited in $80 \%$ of the explants $(n=20)$ by the expression of high amounts of xSpryl (100 pg of RNA per embryo). Injection of $30 \mathrm{pg}$ of xSpryl mRNA or $20 \mathrm{ng}$ of xPAPC antisense morpholino oligonucleotides (MO), which partially blocks translation of the endogenous PAPC mRNA under these conditions (Medina et al. 2004), did not inhibit elongation of the animal cap explants (90\% elongated, $n=20)$. When $20 \mathrm{ng}$ of xPAPC MO were injected together with 30 of pg xSpry1 mRNA, $90 \%$ of the animal caps failed to elongate $(n=20)$. This indicated that reduction of endogenous xPAPC activity enhanced the inhibitory effect of xSpry1 on CE movements. The partial knockdown of xPAPC sensitized the animal caps for the inhibition by $x$ Spryl, supporting the idea of an antagonism between xPAPC and xSpry1 (Supplemental Table S1). This antagonism could be confirmed experimentally in DMZ tissue. Explanted DMZs, so-called Keller explants (Keller 2002), express both PAPC and xSpryl and display CE movements. Elongation was inhibited by overexpression of xSpryl but partially rescued by coexpression of xPAPC or xPAPCc (Fig. 3B). In contrast, xPAPCmut and

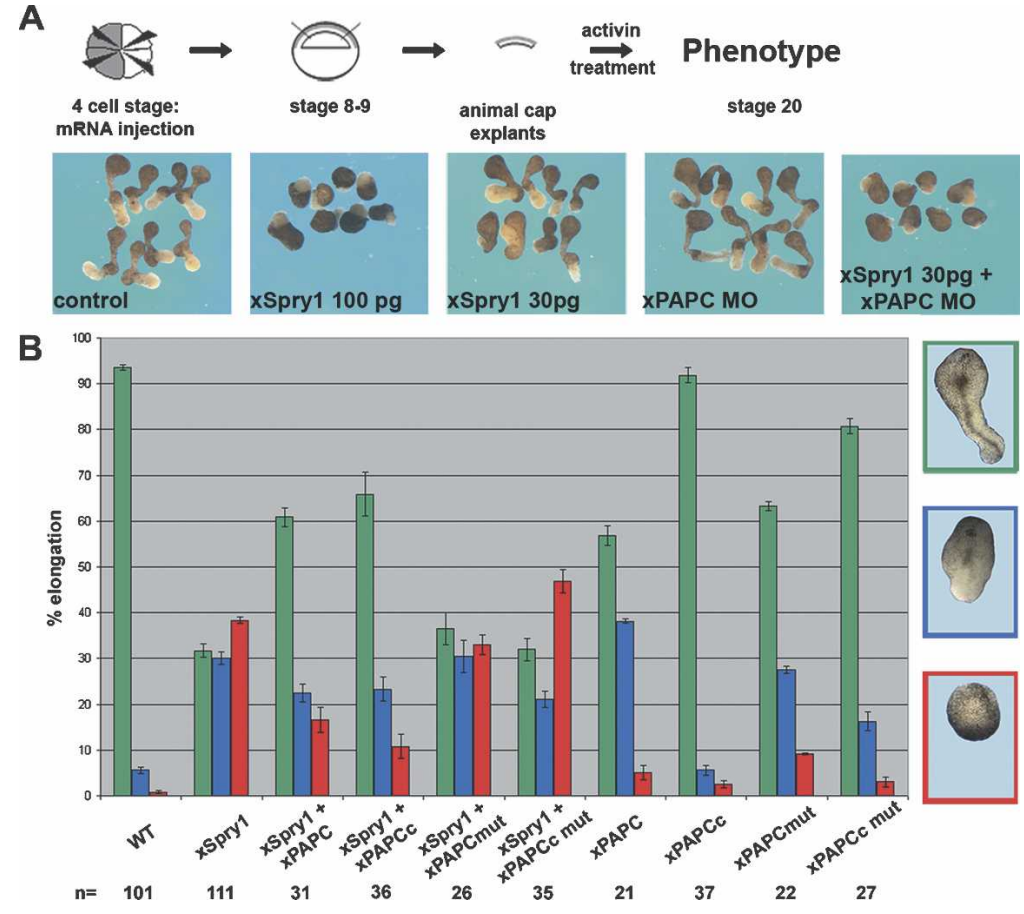

Figure 3. Antagonistic functions of xPAPC and xSpry1 in CE movements. $|A|$ Schematic outline of the animal cap experiment. Synthetic mRNAs were injected into four blastomeres of four-cell-stage embryos. At stage 8.5, animal caps were explanted, exposed to activin for $3 \mathrm{~h}$, and cultured to stage 20. Activin-treated animal caps from control embryos or embryos injected with $100 \mathrm{pg}$ or $30 \mathrm{pg}$ of xSpryl, $20 \mathrm{ng}$ of xPAPC MO, or $30 \mathrm{pg}$ of xSpryl and $20 \mathrm{ng}$ of xPAPC MO. $(B)$ Modulation of CE movements in DMZ explants. mRNAs for xSpryl, xPAPC, xPAPCc, xPAPCmut, and xPAPCcmut (400 pg each) were injected into dorsal blastomeres at the four-cell stage. At stage 10+, DMZs were explanted and the elongation was scored at stage 20. DMZs were grouped in fully elongated (green), partially elongated (blue), and not elongated (red).
xPAPCcmut, which did not interact with xSpryl in yeast and in co-IP experiments and did not induce membrane recruitment of GFP-xSpry, failed to rescue elongation in xPAPC or xPAPCmut inhibited DMZ elongation only weakly, very likely due to the modulation of cadherinmediated adhesion (Chen and Gumbiner 2006). xPAPCc and XPAPCcmut had no adverse effect on DMZ elongaAltogether, these results demonstrate that xPAPC and $x$ Spry 1 have antagonistic activities that contribute to the regulation of CE movements. These data also support the view that the inhibition of xSpry function by $\mathrm{xPAPC}$ is achieved by binding of its intracellular domain to $\mathrm{xSpry}$.

Hallmarks of active PCP signaling are the membrane ocalization of Dishevelled (Dsh) (Wallingford et al. $2000)$ and protein kinase $\mathrm{C} \delta$ (PKC $\delta$ ) (Kinoshita et al. 2003). We therefore examined whether the localization these PCP components could be modulated by the antagonism between xPAPC and xSpry1. GFP-Dsh and GFP-PKC $\delta$ were expressed in Xenopus animal cap cells, ar localization was analyzed by confocal microscopy and quantified (Fig. 4A,B; Supplemental Fig. S4). calization of GFP-Dsh and GFP-PKC $\delta$. This is consistent ith the yeast two-hybrid assay showing that xPAPCc did not bind Dsh (Fig. 1B). The recruitment of Dsh and PKC $\delta$ to the membrane by Fz7 was inhibited by xSpryl. $\mathrm{xPAPC}$ and $\mathrm{xPAPC}$ but not M-PAPC or $\mathrm{xPAPC} c \mathrm{C}_{\mathrm{t}}$ rescued the recruitment of Dsh and PKCס (Fig. 4A,B). Since previous studies have shown that XPAPC can activate RhoA, a downstream effector of the PCP pathway (Medina et al. 2004; Unterseher et al. 2004), we further characterized the antagonism between $\mathrm{xPAPC}$ and $\mathrm{xSpry} 1$ on the level of RhoA activation. Overexpression of xSpryl in Xenopus embryos inhibited RhoA activation. xPAPC but not xPAPCmut antagonized the xSpryl effect and rescued RhoA activation (Fig. 4C). Knockdown of PAPC function by xPAPC MO inhibited RhoA activation (Medina et al. 2004; Unterseher et al. 2004). Importantly, xPAPC but not XPAPCmut could rescue RhoA activation in PAPC-depleted embryos (Fig. 4D). Taken together, these results suggest that $x P A P C$ and $x$ Spry 1 act antagonistically in PCP pathway downstream from $\mathrm{Fz} 7$ and upstream of PKC $\delta$, Dsh, and RhoA. The serine residues 741 and 955 are essential for both xSpryl binding and Rho activation.

In Xenopus, PAPC is able to antagonize xSpry1 activity, and the intracellular domain of xPAPC but not xPAPCmut is able to bind Drosophila sprouty (Fig. 1B; data not shown). Therefore, we used the Drosophila larva as a genetic test system for the inhibition of dSpry by xPAPC. sprouty mutants in Drosophila larvae display enhanced branching of the tracheae and an increased number of terminal cells due to elevated FGF signaling (Hacohen et al. 1998). Therefore, we tested whether xPAPC is able to antagonize Spry in Drosophila larvae, which should result in a higher number of terminal cells. Transgenic fly lines were generated that allow inducible expres- 


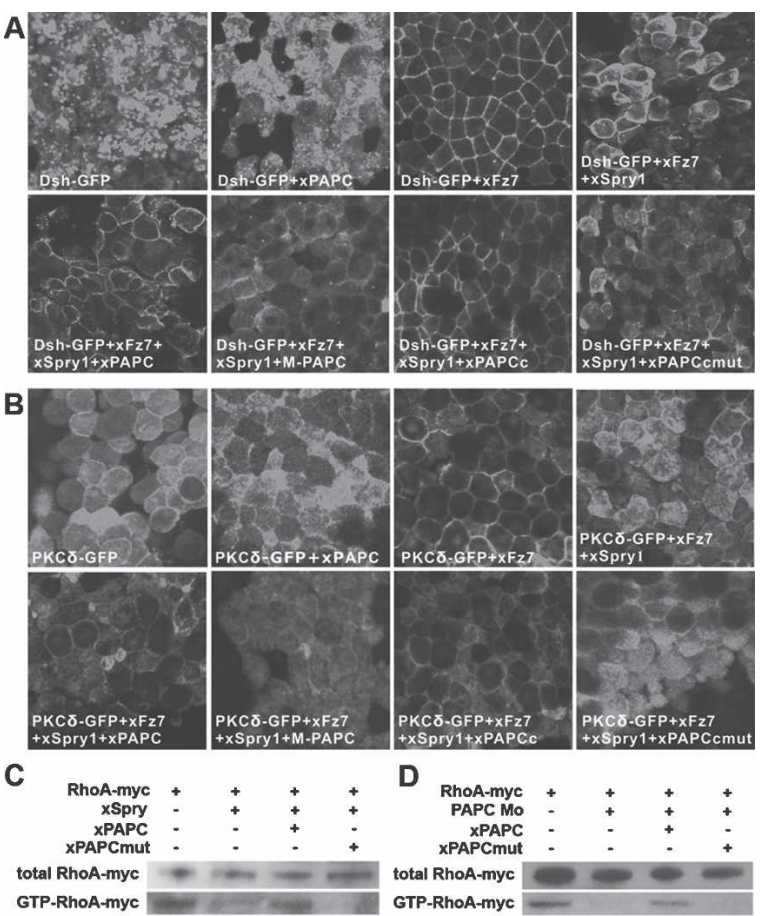

Figure 4. xPAPC stimulates membrane localization of PCP components Dsh and $\mathrm{PKC} \delta$ and activates RhoA by antagonizing xSpryl. $(A, B)$ mRNAs were injected at the four-cell stage, caps were excised at stage 9 , and the localization of Dsh-GFP $(A)$ or PKC $\delta$-GFP $(B)$ was analyzed by confocal microscopy. The amounts of mRNA injected were $300 \mathrm{pg}$ for Dsh-GFP or PKC $\delta$-GFP, $100 \mathrm{pg}$ for Fz7, $1 \mathrm{ng}$ for xSpryl, and $800 \mathrm{pg}$ for xPAPC, M-PAPC, xPAPCc, or xPAPCcmut $(C, D)$ Myc-tagged xRhoA DNA was injected alone or in combination with xSpryl, xPAPC, or xPAPCmut mRNA or xPAPC MO. Activated RhoA was precipitated by GST-RBD and detected on a Western blot with Myc antibody. Whole-embryo lysate was used as loading control.

sion of xPAPC (UAS-PAPC), M-PAPC (UAS-M-PAPC), or XAXPC (UAS-AXPC). Following expression of the protocadherin constructs in the tracheae by breathless-Gal4 (btl-Gal4) (Shiga et al. 1996), the number of terminal cells labeled by GFP was determined for the different genotypes. Wild-type larvae contain two terminal tracheal cells per segment (Fig. 5F), but three terminal cells were seen in tracheae in which FGF signaling was elevated by expression of an activated allele of FGF receptor (UAS- $\lambda$-btl) (Fig. 5A; Lee et al. 1996). Expression of PAPC in wild-type larvae $\left(\right.$ Spry $\left.^{+/+}\right)$caused a higher number of terminal cells. About $30 \%$ of larvae had three or four terminal cells in at least one segment (Fig. 5B; Supplemental Fig. S5). This phenotype was rarely seen for the other genotypes (expression of M-PAPC or AXPC) (Fig. 5C,D; Supplemental Fig. S5). To test whether the effect of PAPC is based on an antagonism of Spry, we reduced the gene dose of spry to $50 \%$. The percentage of larvae with segments containing more than two terminal cells increased to $>60 \%$ (Supplemental Fig. S5). Since no marked chromosomes were used in this cross we could not discriminate between wild-type and Spry heterozygous larvae. To quantify the genetic interaction of PAPC and Spry more precisely, we used a more stringent way of analysis and employed marked chromosomes for this cross. This allowed us to score the $s p r y^{+/+}$and spry $^{+/-}$larvae separately. We determined the percentage of larval segments showing three or more terminal cells (120 segments counted = 15 larvae). In spry heterozygous larvae, we found that three to five terminal cells were present in $38 \%$ of all segments that expressed FL-PAPC (Fig. 5E). In contrast, $4 \%-8 \%$ of larval segments expressing M-PAPC and AXPC had more than two terminal cells (Fig. 5E). This experiment demonstrated that the higher number of terminal cells seen upon expression of PAPC was enhanced when in addition the gene dose of spry was reduced. It supports the idea that PAPC sequesters Spry and thereby antagonizes its function. This inhibition is specific because AXPC is unable to do so. The M-PAPC mutant lacking $C$ terminus cannot antagonize Spry, indicating that the cytoplasmic domain of xPAPC is crucial for this function. Since there is no PAPC homolog in Drosophila, one can conclude from these experiments that the domain of dSpry protein that is essential for the interaction with PAPC is conserved between Xenopus and Drosophila for reasons other than PAPC interaction. The experiments in Xenopus and Drosophila also demonstrated that PAPC is able to antagonize the different functions of Spry. The inhibitory effects of Spry on PCP in Xenopus as well as on MAPK pathway in Drosophila could be counteracted by xPAPC.

In Xenopus, Spry inhibits the PCP pathway in the cytoplasm via an unknown mechanism. As a result, Dsh and PKC $\delta$ are not localized to the plasma membrane and RhoA is not activated. Recently, it was reported that Xenopus ANR5 protein, which can modulate gastrulation movements, interacts with xPAPC, corroborating the role of PAPC in intracellular signaling (Chung et al. 2007). Our results presented here show that gastrulation movements can be modulated by sequestration of the inhibitor Spry by the cytoplasmic domain of xPAPC. This mechanism provides a direct link between protocadherins and the PCP pathway and contributes a novel regulatory module to $\beta$-catenin-independent Wnt signaling.

\section{Materials and methods}

\section{DNA constructs}

For the yeast two-hybrid assay, the cytoplasmic domain of xPAPC (residues 715-979) was amplified by PCR and cloned into the LexA-based bait plasmid pNLX3 to generate pNLX3-xPAPCc. xSpry2, xSpred1, or xDsh was cloned into the Gal4-AD-based prey plasmid pACT2 to generate pACT2-xSpry2, pACT2-xSpred1, or pACT2-xDsh. For expression in Xenopus, the cytoplasmic domain of xPAPC or XAXPC (residues 8381016) was cloned into pCS2 + Flag to generate Flag-xPAPCc or Flag-xAXPCc. Mutant constructs pNLX3-xPAPCc-S741A, pNLX3-xPAPCcS955A, pNLX3-xPAPCc-S741E, pNLX3-xPAPCc-S955E, Flag-xPAPCcS741A/S955A (xPAPCcmut), and xPAPC-S741A/S955A (xPAPCmut) were generated by PCR site-directed mutagenesis. All of the engineered constructs were confirmed by sequencing. Myc-xSpryl was kindly provided by E. Nishida (Hanafusa et al. 2002).

\section{Xenopus embryo manipulation}

xPAPC antisense MOs MoPAPC I and II (1:1), which target both xPAPC alleles, were injected (Medina et al. 2004). Capped mRNAs were synthesized from linearized plasmids using the mMessage mMachine Kit (Ambion). In vitro fertilization, embryo culture and staging, microinjections, and Keller explants were performed as described (Nieuwkoop and Faber 1975; Medina et al. 2004; Unterseher et al. 2004). For confocal microscopy, animal caps were excised at stage 9 , fixed in $4 \%$ formaldehyde, and subjected to microscopic analysis using a Nikon C1Si spectral imaging confocal laser scanning system on a Nikon TE2000-E inverted microscope. 

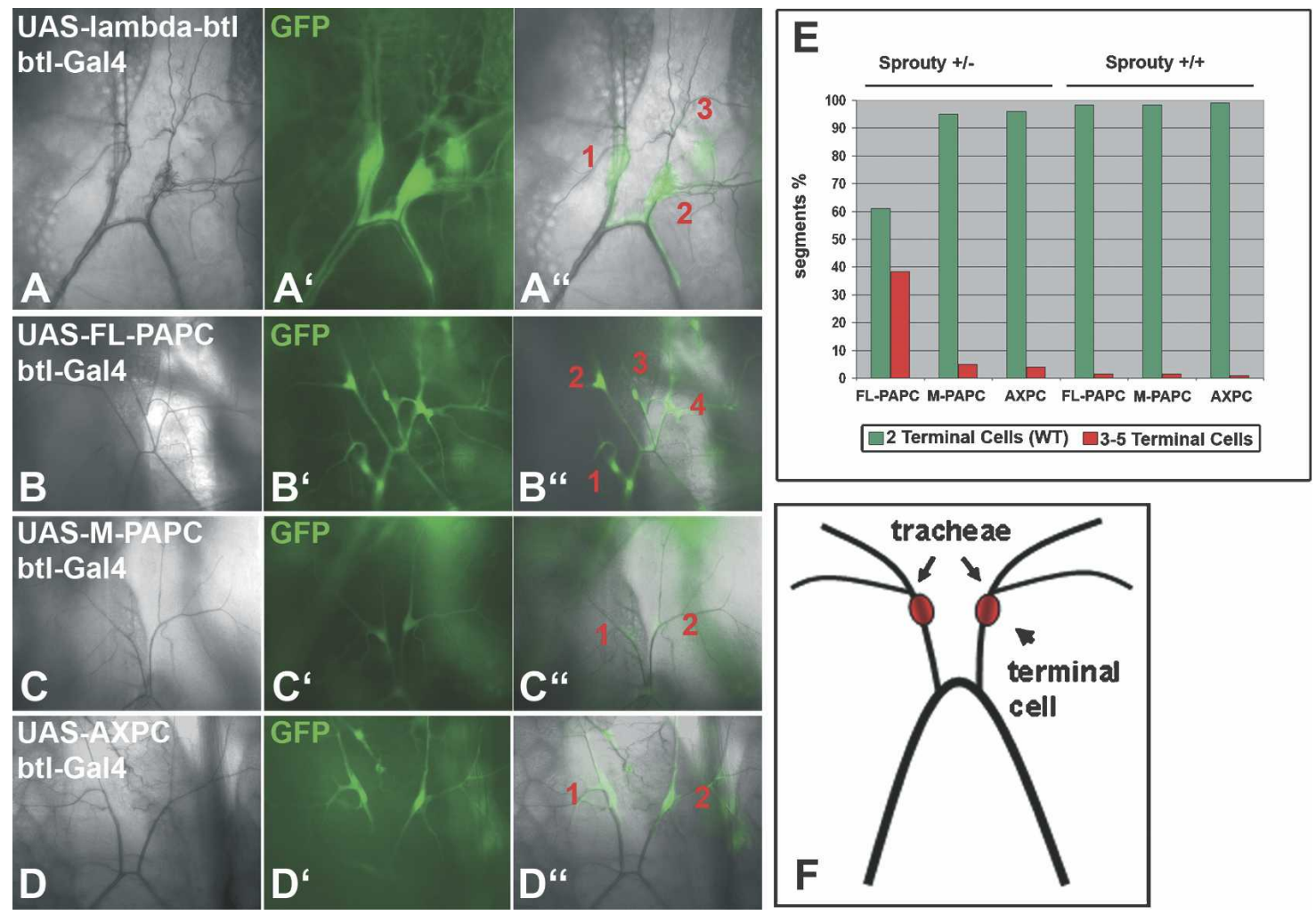

Figure 5. xPAPC antagonizes dSpry in the tracheae of Drosophila larvae. Btl-Gal4 flies were crossed with UAS- $\lambda$-btl $(A)$, UAS full-length (FL) PAPC $(B)$, UAS-M-PAPC $(C)$, and UAS-AXPC $(D)$. Morphology of the tracheae and number of terminal cells were analyzed in third instar larvae. $(A-D)$ Bright-field microscopy. $\left(A^{\prime}-D^{\prime}\right)$ GFP fluorescence visualising the trachea. $\left(A^{\prime \prime}-D^{\prime \prime}\right)$ Overlay of GFP and bright-field. $(E)$ Analysis of terminal cells in segments of larvae heterozygous for spry (sty[266]) expressing FL-PAPC, M-PAPC, or AXPX in the trachea. (F) Schematic drawing of trachea and terminal cells in one larval segment.

Whole-mount in situ hybridization

xPAPC mRNA was detected in gastrula embryos as described in Medina et al. (2004). The digoxigenin-labeled xSpry antisense RNA probe was synthesized by T7 RNA polymerase from the Myc-xSpryl plasmid linearized with HindIII.

\section{Cell culture and immunofluorescence}

Transfection of HEK293 cells was performed with linear polyethylenimine MW $\sim 25,000$ (Polysciences, Inc.). After transfection cells were grown in DMEM Ready Mix or serum-free DMEM high-glucose medium (both from PAA Laboratories) for $18 \mathrm{~h}$. Medium was then exchanged for fresh DMEM Ready Mix, serum-free medium, or serum-free medium containing $5 \mathrm{ng} / \mathrm{mL}$ bFGF (Invitrogen) and $0.1 \mathrm{mg} / \mathrm{mL}$ BSA or $5 \mu \mathrm{M}$ SU5402 (Calbiochem) or both. Cells were washed with ice-cold PBS and fixed in $4 \%$ formaldehyde. For immunofluorescence, cells were stained with $\alpha$-PAPC $11 \mathrm{~A} 6 \mathrm{mAb}$ (1:100) (kindly provided by B.M. Gumbiner) and $\alpha$-mouse-Alexa488 Ab (1:500) (Molecular Probes). Fluorescent images were acquired using a Nikon Eclipse 80i upright microscope, a Nikon DS-Qi1Mc CCD camera, and the NIS-Elements 2.30 program.

Yeast two-hybrid assay

For the two-hybrid screen, the yeast strain L40 was cotransformed with pNLX3-xPAPCc and a $X$. laevis oocyte cDNA library (Matchmaker, Clontech) by standard protocols. Positive clones were selected on medium lacking leucine, tryptophan, and histidine and were checked with a filter assay for $\beta$-galactosidase activity. Prey plasmids were then isolated and retransformed into yeast with pNLX3-xPAPCc or with pNLX3 as a negative control to confirm the specificity of the interaction.

Co-IP

Xenopus embryos were coinjected with 500 pg of Myc-xSpryl and $500 \mathrm{pg}$ of Flag-xPAPCc, Flag-xPAPCcmut, or Flag-xAXPCc mRNA at the fourcell stage. Protein was extracted from embryos grown to gastrula stage and subjected to immunoprecipitation as described (Medina et al. 2004). Antibodies used were $\alpha$-Myc Ab-1 (Oncogene) and $\alpha$-Flag M2 (Sigma).

Phosphatase treatment of protein extracts

Lysates from gastrula-stage embryos were immunoprecipitated with $\alpha$ Flag $\mathrm{Ab}$, resuspended in $\lambda$ protein phosphatase buffer, and incubated for $1.5 \mathrm{~h}$ at $30^{\circ} \mathrm{C}$ in the presence or absence of $\lambda$ protein phosphatase. FlagxPAPCc and Flag-xPAPCcmut were detected on a Western blot with the Flag $\mathrm{Ab}$

\section{RhoA activity assay}

Xenopus embryos were injected in the animal pole at the four-cell stage with Myc-tagged xRhoA DNA (50 pg) alone or in combination with 600 $\mathrm{pg}$ of xSpryl, $40 \mathrm{ng}$ of $\mathrm{xPAPC} \mathrm{MO}, 800 \mathrm{pg}$ of $\mathrm{xPAPC}$, or $800 \mathrm{pg}$ of xPAPCmut mRNA. Protein was extracted from 40 embryos grown to stage 10.5 and subjected to GST-RBD-binding assay as described (Medina et al. 2004; Kitzing et al. 2007).

\section{Experiments with Drosophila}

Constructs and GFP to mark the trachea were expressed from UAS transgenes with breathless-Gal4 (btl-Gal4). spry heterozygous larvae were recognized by the absence of the segregating balancer chromosome marked with a uniformly expressed actin-GFP transgene. $\lambda$-btl encodes a constitutively active btl protein. For analysis of trachea morphology, third instar larvae, raised at $29^{\circ} \mathrm{C}$, were anethetized on ice, mounted in halocarbon oil, and photographed with bright-field and fluorescence optics. The number of terminal cells in each segment of 15 larvae per genotype was counted. The following stocks were used: UAS- $\lambda$-btl (III. chr.), btlGal4, UAS-GFP (II. chr.), and sty[226] (obtained from S. Luschnig, Bayreuth). PAPC, M-PAPC, and AXPC were cloned from pCS plasmids into corresponding sites of the vector pUAST. 


\section{Acknowledgments}

We thank O. Staub for providing the yeast two-hybrid cDNA library, E. Amaya and E. Nishida for constructs, B. Gumbiner for antibodies, R. Grosse for GST-RBD protein, M. Boutros for Drosophila experiments, and $\mathrm{K}$. Linsmeier for expert technical assistance. We are grateful to $\mathrm{S}$. Cramton for critically reading the manuscript. The confocal images were acquired at the Nikon Imaging Center at the University Heidelberg. This work was supported by a research grant of the Deutsche Forschungsgemeinschaft (STE-613/4-1).

\section{References}

Chen, X. and Gumbiner, B.M. 2006. Paraxial protocadherin mediates cell sorting and tissue morphogenesis by regulating $\mathrm{C}$-cadherin adhesion activity. J. Cell Biol. 174: 301-313.

Chung, H.A., Yamamoto, T.S., and Ueno, N. 2007. ANR5, an FGF target gene product, regulates gastrulation in Xenopus. Curr. Biol. 17: 932 939.

Hacohen, N., Kramer, S., Sutherland, D., Hiromi, Y., and Krasnow, M.A 1998. sprouty encodes a novel antagonist of FGF signaling that patterns apical branching of the Drosophila airways. Cell 92: 253-263.

Hanafusa, H., Torii, S., Yasunaga, T., and Nishida, E. 2002. Sprouty1 and Sprouty2 provide a control mechanism for the Ras/MAPK signalling pathway. Nat. Cell Biol. 4: 850-858.

Hukriede, N.A., Tsang, T.E., Habas, R., Khoo, P.L., Steiner, K., Weeks, D.L., Tam, P.P., and Dawid, I.B. 2003. Conserved requirement of Lim1 function for cell movements during gastrulation. Dev. Cell 4: 83-94.

Keller, R. 2002. Shaping the vertebrate body plan by polarized embryonic cell movements. Science 298: 1950-1954.

Kim, S.H., Yamamoto, A., Bouwmeester, T., Agius, E., and De Robertis, E.M. 1998. The role of paraxial protocadherin in selective adhesion and cell movements of the mesoderm during Xenopus gastrulation. Development 125: 4681-4690.

Kinoshita, N., Iioka, H., Miyakoshi, A., and Ueno, N. 2003. PKC $\delta$ is essential for Dishevelled function in a noncanonical Wnt pathway that regulates Xenopus convergent extension movements. Genes \& Dev. 17: 1663-1676.

Kitzing, T.M., Sahadevan, A.S., Brandt, D.T., Knieling, H., Hannemann, S., Fackler, O.T., Grosshans, J., and Grosse, R. 2007. Positive feedback between Dial, LARG, and RhoA regulates cell morphology and invasion. Genes \& Dev. 21: 1478-1483.

Lee, T., Hacohen, N., Krasnow, M., and Montell, D.J. 1996. Regulated Breathless receptor tyrosine kinase activity required to pattern cell migration and branching in the Drosophila tracheal system. Genes \& Dev. 10: 2912-2921.

Mason, J.M., Morrison, D.J., Basson, M.A., and Licht, J.D. 2006. Sprouty proteins: Multifaceted negative-feedback regulators of receptor tyrosine kinase signaling. Trends Cell Biol. 16: 45-54.

Medina, A., Swain, R.K., Kuerner, K.M., and Steinbeisser, H. 2004. Xenopus paraxial protocadherin has signaling functions and is involved in tissue separation. EMBO I. 23: 3249-3258.

Nieuwkoop, P.D. and Faber, J. 1975. Normal table of Xenopus laevis (Daudin). North Holland Publishing Company, Amsterdam, The Netherlands.

Nutt, S.L., Dingwell, K.S., Holt, C.E., and Amaya, E. 2001. Xenopus Sprouty2 inhibits FGF-mediated gastrulation movements but does not affect mesoderm induction and patterning. Genes \& Dev. 15: 1152-1166.

Schambony, A. and Wedlich, D. 2007. Wnt-5A/Ror2 regulate expression of XPAPC through an alternative noncanonical signaling pathway. Dev. Cell 12: 779-792.

Shiga, Y., Tanaka-Matakatsu, M., and Hayashi, S. 1996. A nuclear GFP/ $\beta$-galactosidase fusion protein as a marker for morphogenesis in living Drosophila. Dev. Growth Differ. 38: 99-106.

Sivak, J.M., Petersen, L.F., and Amaya, E. 2005. FGF signal interpretation is directed by Sprouty and Spred proteins during mesoderm formation. Dev. Cell 8: 689-701.

Unterseher, F., Hefele, J.A., Giehl, K., De Robertis, E.M., Wedlich, D., and Schambony, A. 2004. Paraxial protocadherin coordinates cell polarity during convergent extension via Rho A and JNK. EMBO J. 23: 3259-3269.

Wallingford, J.B., Rowning, B.A., Vogeli, K.M., Rothbacher, U., Fraser,
S.E., and Harland, R.M. 2000. Dishevelled controls cell polarity during Xenopus gastrulation. Nature 405: 81-85.

Wessely, O., Kim, J.I., Geissert, D., Tran, U., and De Robertis, E.M. 2004. Analysis of Spemann organizer formation in Xenopus embryos by cDNA macroarrays. Dev. Biol. 269: 552-566.

Yamamoto, A., Amacher, S.L., Kim, S.H., Geissert, D., Kimmel, C.B., and De Robertis, E.M. 1998. Zebrafish paraxial protocadherin is a downstream target of spadetail involved in morphogenesis of gastrula mesoderm. Development 125: 3389-3397. 


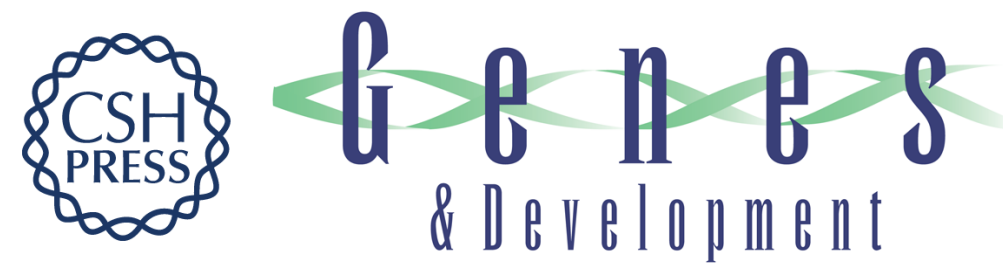

\section{Xenopus Paraxial Protocadherin regulates morphogenesis by antagonizing Sprouty}

Yingqun Wang, Patricia Janicki, Isabelle Köster, et al.

Genes Dev. 2008, 22:

Access the most recent version at doi:10.1101/gad.452908

Supplemental http://genesdev.cshlp.org/content/suppl/2008/03/12/22.7.878.DC1
Material

References This article cites 20 articles, 9 of which can be accessed free at:

http://genesdev.cshlp.org/content/22/7/878.full.html\#ref-list-1

License

Email Alerting Receive free email alerts when new articles cite this article - sign up in the box at the top

Service right corner of the article or click here.

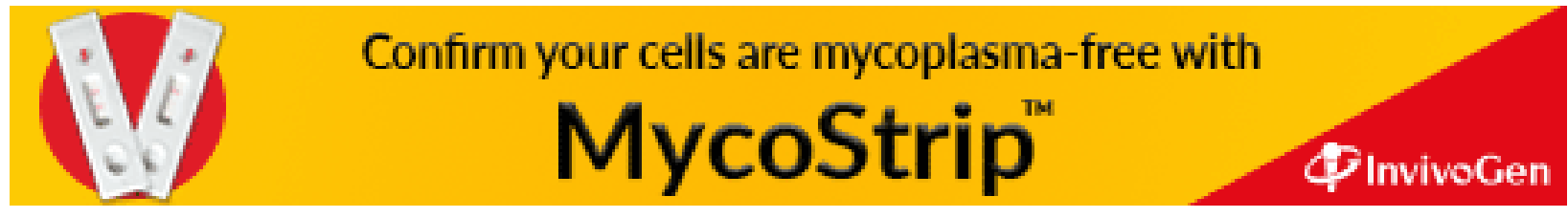

